\title{
Emerging Roles of Wild-type and Mutant IDH1 in Growth, Metabolism and Therapeutics of Glioma
}

\author{
Matthew Garrett ${ }^{1} \bullet$ Yuki Fujii ${ }^{2,3} \bullet$ Natsuki Osaka $^{4} \bullet$ Doshun Ito $^{5} \bullet$ \\ Yoshihisa Hirota ${ }^{2,6} \bullet$ Atsuo T. Sasaki ${ }^{1,2,4,7}$ \\ ${ }^{1}$ Department of Neurosurgery, Brain Tumor Center at UC Gardner Neuroscience Institute, \\ Cincinnati, OH, USA; ${ }^{2}$ Division of Hematology and Oncology, Department of Internal \\ Medicine, University of Cincinnati College of Medicine, Cincinnati, OH, USA; ${ }^{3}$ Graduate \\ School of Science, Osaka City University, Sugimoto, Sumiyoshi, Osaka, Japan; ${ }^{4}$ Institute for \\ Advanced Biosciences, Keio University, Tsuruoka, Japan; ${ }^{5}$ Structural Biology Research \\ Center, Institute of Materials Structure Science, High Energy Accelerator Research \\ Organization (KEK), Tsukuba, Ibaraki, Japan; ${ }^{6}$ Department of Bioscience and Engineering, \\ College of Systems Engineering and Science, Shibaura Institute of Technology, Fukasaku, \\ Minuma-ku, Saitama, Japan; ${ }^{7}$ Department of Cancer Biology, University of Cincinnati \\ College of Medicine, OH, USA.
}

Author for correspondence: Atsuo T. Sasaki, Division of Hematology and Oncology, Department of Internal Medicine, University of Cincinnati College of Medicine, Cincinnati, OH, USA. Email: atsuo.sasaki@uc.edu

Doi: https://doi.org/10.36255/exonpublications.gliomas.2021.chapter4

\begin{abstract}
Glioblastoma is one of the most devastating human malignancies and is categorized into primary and secondary glioblastoma subtypes that develop through different genetic pathways. Isocitrate dehydrogenase 1 (IDH1) and 2 (IDH2) are key enzymes linking cellular metabolism to epigenetic regulation and redox states. Hot spot mutations of IDH1 is early and frequent genetic alterations in secondary glioblastoma as well as in grade II and III glioma and represent a major biomarker with diagnostic, prognostic, and predictive implications. Mutant IDH proteins acquire neomorphic enzymatic activity to produce D-2hydroxyglutarate, a putative oncometabolite that could induce epigenetic changes
\end{abstract}

In: Gliomas. Debinski W (Editor). Exon Publications, Brisbane, Australia. ISBN: 978-0-6450017-4-7; Doi: https://doi.org/10.36255/exonpublications.gliomas.2021

Copyright: The Authors.

License: This open access article is licenced under Creative Commons Attribution-NonCommercial 4.0 International (CC BY-NC 4.0) https://creativecommons.org/licenses/by-nc/4.0/ 
at DNA and RNA levels. On the other hand, recent studies show that primary glioblastoma increases expression of wild-type IDH1, which confers therapeutic resistance. In this chapter, we introduce the current understanding of the biological roles of wild-type and mutant IDH enzymes in glioblastoma. We discuss the challenges hampering the development of IDH targeted therapeutics and the current status of IDH1 mutant inhibitor development.

Keywords: glioblastoma; glioma; IDH1; metabolism; therapeutics

\section{INTRODUCTION}

Gliomas are the most common primary malignant brain tumor in adults. Many WHO grade I gliomas are well-circumscribed, surgically curable tumors and have different molecular drivers than those seen in grade II, III, and IV gliomas. Even though grade II glioma is categorized as a low-grade glioma, it is incurable due to its diffusely infiltrative nature and that it almost inevitably progress to high-grade III glioma and grade IV secondary glioblastoma over time (1). Remarkably, over $70 \%$ of grade II gliomas and secondary glioblastoma possess heterozygous missense mutations in the gene encoding cytosolic enzyme IDH1 (2-4), which confer a neomorphic enzyme activity that converts $\alpha$-ketoglutarate $(\alpha \mathrm{KG})$ to D-2hydroxyglutarate (D-2HG) (Figure 1) (5). Mutations in mitochondrial isozyme IDH2 have also been identified in gliomas, but they are much less common and mutually exclusive with mutations in $\operatorname{IDH1}(3,6,7)$. Though naturally existing $\mathrm{D}-2 \mathrm{HG}$ is at negligible levels, the intracellular concentrations of D-2HG reach 10-30 mM in the glioma with the IDH1 mutation (5). D-2HG appears to be a major intracellular effector of IDH1 mutated glioma and is considered as an oncometabolite, altering epigenetics and setting the cellular state permissive to malignant transformation (8-10).

There are three distinct groups of gliomas with different molecular drivers, mutations, epigenetic signatures, and clinical behavior: (i) IDH wild-type gliomas (primary glioblastoma); (ii) IDH mutant with a 1p/19q deletion; and (iii) IDH mutant with a p53 mutation (11). This distinction between the three groups of

Isocitrate

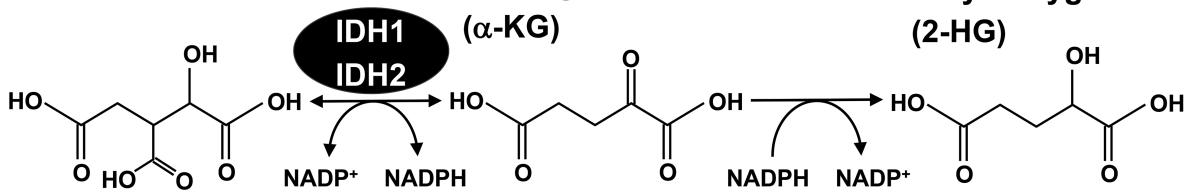

Figure 1. Wild-type IDH converts isocitrate and $\alpha$-ketoglutarate to each other, and mutant IDH converts $\boldsymbol{\alpha}$-ketoglutarate to 2-hydroxyglutarate. Isocitrate dehydrogenase (IDH) interconverts isocitrate and $\alpha$-ketoglutarate. Three subtypes of human IDH are known: IDH1 (cytosolic, $\mathrm{NADP}^{+}$dependent) and IDH2 (mitochondrial, NADP ${ }^{+}$dependent) and IDH3 (mitochondrial, $\mathrm{NAD}^{+}$dependent). IDH1 and IDH2 mutations have been reported in a variety of cancers such as, glioma, acute myeloid leukemia and bile duct cancer. Mutated IDH converts $\alpha$-ketoglutarate to 2-hydroxyglutarate, which worsens the prognosis of gliomas. IHD1 and IDH2 mutations produce D-2-hydroxyglutarate, which has been considered "oncometabolite." 
gliomas is currently driving clinical management as well as placing greater emphasis on the molecular and genetic differences of IDH mutant and IDH wild-type gliomas. This chapter discusses the role of wild-type and mutant IDH1 enzymes in the progression of glioma, and emerging therapy targeting the glioma with wild-type or mutant IDHl.

\section{METABOLIC CHARACTERIZATION OF WILD-TYPE IDH1 GLIOMA}

There are three isocitrate dehydrogenase isozymes-IDH1, IDH2, and IDH3that are expressed in mammalian cells. IDH1 is a cytosolic enzyme, while IDH2 and IDH3 are mitochondrial enzymes. Both IDH1 and IDH2 use $\mathrm{NADP}^{+}$as an electron acceptor to convert isocitrate to $\alpha \mathrm{KG}$, co-producing an NADPH per reaction. IDH3 uses $\mathrm{NAD}^{+}$as an electron acceptor. Notably, the ratios of NADPH/ $\mathrm{NADP}^{+}$determine the intracellular redox potential, affecting the thermodynamic driving force of many reactions, in particular providing electrons for lipids and deoxyribonucleotide and reducing oxidized precursors to maintain a reduced intracellular condition and ameliorate oxidative damage (Figure 2). The IDH1 and IDH2-dependent reaction is reversible, while IDH3-dependent reaction is

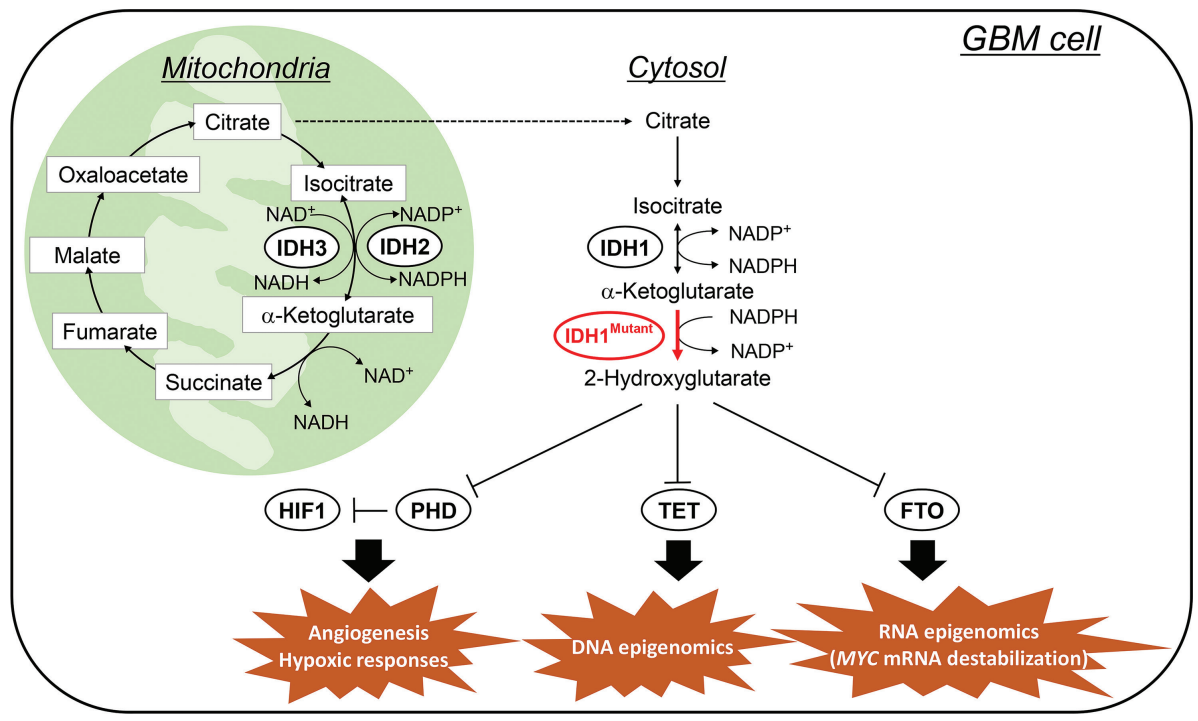

Figure 2. D-2HG generated by IDH1 mutation interferes various pathways resulting in glioblastoma exacerbation. Citrate, the mitochondrial metabolite, flows out to cytosol. Isocitrate, synthesized by cytosolic citrate, is used as a substrate for the IDH1-mediated catabolism. The mutant IDH1 produces D-2-hydroxyglutarate (D-2HG) as an oncometabolite. In glioblastoma, accumulated D-2HG causes (i) angiogenesis and hypoxic responses through depression of HIF1 by PHD inhibition; (ii) reprograming of DNA epigenomics through TET inhibition; and (iii) reprograming of RNA epigenomics including destabilization of MYC mRNA through FTO inhibition. D-2HG, 2-hydroxyglutarate; FTO, fat mass and obesityassociated protein; HIF1, hypoxia inducible factor 1 ; IDH1, isocitrate dehydrogenase $1 ;$ PHD, prolyl hydroxylase domain -containing protein (PHD); TET, ten-eleven translocation enzyme. 
irreversible (12-14). The reversible nature of IDH1 and IDH2 reaction plays an important role in reductive carboxylation, which enables cells lipogenesis under the conditions that decrease the TCA cycle coupled-oxidative phosphorylation (for example, hypoxia, VHL mutation) $(15,16)$ (Figures 1-3).

\section{Wild-type IDH1 is overexpressed in many primary glioblastoma}

Although wild-type IDH1 has had much less attention compared to the research on glioma with IDH1 mutation, several studies have revealed that wild-type IDH1 is overexpressed in several types of cancers, including non-small cell lung carcinoma (NSCLC) $(17,18)$, pancreatic adenocarcinoma (PDAC) (19), and primary glioblastoma $(20,21)$. Importantly, these studies show that IDHl is overexpressed in over $60 \%$ of primary glioblastoma patients and is correlated with poor overall survival. Wahl et al. and Calvert et al. independently demonstrated that knocking down endogenous IDH1 by shRNA, or pharmacological inactivation of IDH1 by the IDH1 inhibitor GSK864, decreases glioblastoma growth in vitro and extends survival of mice harboring intracranial glioblastoma, while overexpression of wild-type IDH1 shortened the survival of the glioblastoma mouse $(20,21)$.

\section{Targeting the wild-type IDH to increase the therapeutic efficacy of radiation and chemotherapies}

With regard to the primary glioblastoma, even with aggressive multimodal radiation and chemotherapy after surgery, only marginal improvements on survival are made (average of 2 months), with a median survival of just 14.6 months $(22,23)$. The use of tumor treating fields (TTFs) with the standard of care therapy in glioblastoma (IR plus TMZ) in a randomized open-label trial of 695 glioblastoma patients, reporting that median progression-free survival was 6.7 months in the TTF plus standard of care group versus 4 months in the standard of care group alone (24). Recent studies suggest that the IDH1 enzyme is a potential clinical target for glioblastoma therapy (25). The rationale is that IDHl activity is considered to increase cytoplasmic NADPH/NADP ${ }^{+}$ratios, which promotes lipid biosynthesis and increases cellular defense against oxidative stress. Suppression of IDHl activity could alter cellular metabolism, potentially lowering the ratio of $\mathrm{NADPH} / \mathrm{NADP}^{+}$, which sensitizes cells to oxidative stresses (Figure 3). Given that radiotherapy induces cell death through induction of reactive oxygen species (ROS) and DNA lesions $(26,27)$, targeting the IDHl enzyme in glioma with wild-type IDH1 allele (for example, primary glioblastoma) has exciting therapeutic potential.

Wahl et al. further demonstrated that knocking down wild-type IDH1 in primary glioblastoma cell lines (wild-type IDH1) decreases the ratio of NADPH/ $\mathrm{NADP}^{+}$, as well as levels of deoxynucleotides and reduced glutathione (GSH) and increases the efficacy of radiation. This radiosensitization effect of IDHl knockdown is reversed by treatment of anti-oxidant $\mathrm{N}$-acetyl cysteine and/or nucleotide precursors, pointing that IDH1-dependent NADPH production is critical for glioblastoma radioresistance $(20,28)$. Likewise, suppression of mitochondrial IDH2 also increases radiosensitivity of primary glioblastoma cells (29). Furthermore, IDH1 inhibition decreases GSH and NADPH levels in the glioblastoma initiating 


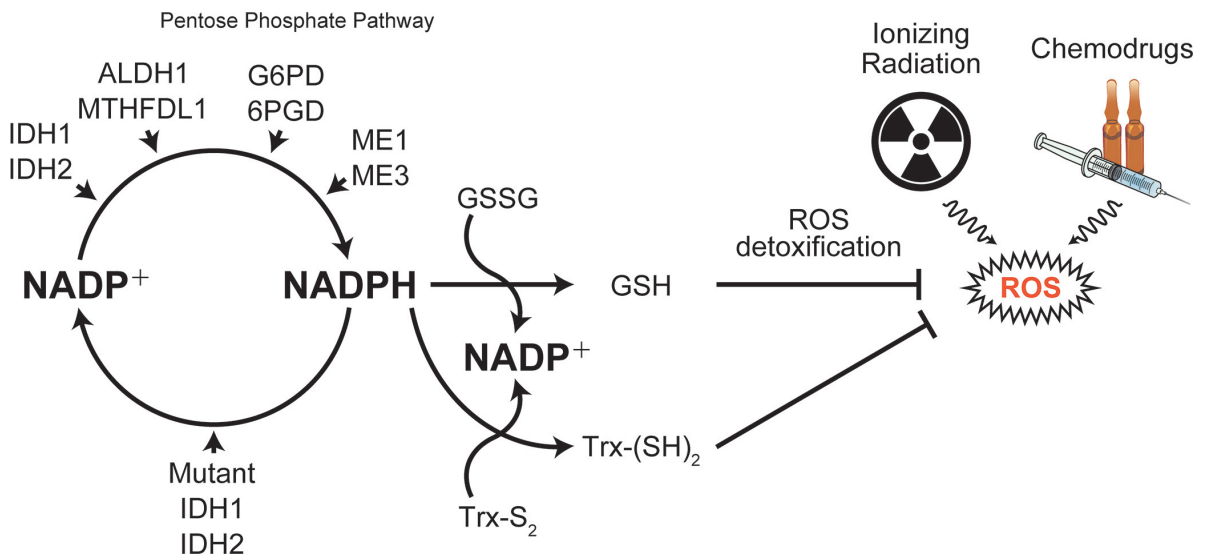

Figure 3. Effects of wild-type and mutant IDH enzymes on redox status and therapeutic efficacy. IDHs mutation may increase therapeutic efficacy of radiation and chemotherapies. IDH1, 2, and the other enzymes reduce NADP+ to NADPH. NADPH also reduces GSSG or Trx- $\mathrm{S}_{2}$ to GSH or $\operatorname{Trx}-(\mathrm{SH})_{2}$, respectively. GSH and Trx- $(\mathrm{SH})_{2}$ detoxify ROS and decrease the effect of the radiation or chemotherapy treatment for glioblastoma patients. The decrease of NADPH/NADP ${ }^{+}$ratio by the inhibition of IDH enzymes has potential to increase the efficacy of the current treatment for glioblastoma. 6PGD, 6-Phosphogluconate dehydrogenase; ALDH1, aldehyde dehydrogenase; G6PD, glucose-6-phosphate dehydrogenase; GSH, glutathione; GSSG, glutathione disulfide; $\mathrm{IDH}$, isocitrate dehydrogenase; $\mathrm{ME}$, malic enzyme; NADP ${ }^{+} / \mathrm{NADPH}$, nicotinamide adenine dinucleotide phosphate; ROS, reactive oxygen species; Trx, thioredoxin.

cells/glioblastoma stem cells carrying EGFR-amplification, making them more susceptible to EGFR inhibitor treatment (30). Serum-free culturing techniques have revealed a sub-population of "glioblastoma initiating cells" that may have increased radiation resistance and lead to recurrence after radiation treatment (31-34). The possibility of better targeting these cells via IDH1 inhibition may lead to better radiation response and delayed recurrence (22-24).

\section{BIOLOGICAL IMPACT OF D-2HG ON GLIOBLASTOMA}

A point mutation in the IDH1 gene was initially identified through exome sequencing of colon tumor and glioblastoma samples $(2,35)$. Mutations in IDH1 mostly occur at Arg-132 residue (R132) located within the catalytic domain, which is the binding site for isocitrate. $\mathrm{R} 132 \mathrm{H}$ is the most common alteration, comprising $>80 \%$ of all IDH1 mutations in gliomas. Surprisingly, a study undertaking metabolomics analysis shows that the mutation of IDH enzymes bestows a new enzymatic function of reducing alpha-ketoglutarate $(\alpha \mathrm{KG})$ to D-2-hydroxyglutarate (D-2HG, or R-2HG) using NADPH as an electron donor (5). In the presence of the IDH1 mutation, the D-2HG molecule, which is normally found at minute levels, can increase to millimolar amounts (5). D-2HG generated by IDH1 mutation interferes various pathways resulting in glioblastoma exacerbation (Figure 2). Understandably, there has been considerable interest in what role this potential new "oncometabolite" might have on cells. Following the discovery of the IDH1 mutation, many investigators sought to determine what new malignant traits this 
mutation would bestow upon a cell. Unexpectedly, it appeared that in general, the addition of the IDH1 mutation led to slower growth in most brain tumor models (36). This was a perplexing result and was hypothesized to be the reason why IDH1 mutant tumors had a better prognosis compared to glioblastomas without the IDH1 mutation. Extensive studies have shown the complexity of the IDHI mutational effects and important issues affecting the interpretation of past research. The impact of D-2HG on transcriptional landscape, with particular emphasis on the recent discovery of the new effect of D-2HG on RNA epigenomics, is discussed below.

\section{D-2HG induces epigenetic alternations by increased DNA and histone methylation}

Even though mechanistic understanding of IDH mutations and D-2HG effects on gliomagenesis remain to be clarified, compelling evidence from Turcan et al. shows that the glioma-associated IDH mutation promotes hypermethylation of histone and DNA through its accumulated product D-2HG (37) (Figure 2). Mechanistically, because of its structural similarity to $\alpha \mathrm{KG}$, it has been considered that supra-physiologically elevated D-2HG levels inhibit enzymes, such as DNA demethylase ten-eleven translocation enzymes (TETs) and histone lysine demethylases (KDMs) that utilize $\alpha \mathrm{KG}$ as a co-substrate (Figure 2). Consequently, IDH1 mutation provokes epigenetic reprogramming of the transcriptional landscape of glioma $(8,38-41)$.

\section{D-2HG inhibits RNA demethylase, FTO, leading to aberrant RNA methylation and growth suppression}

While the dynamic covalent modifications (for example, methylation) to DNA and histones play critical roles in regulating gene transcriptions, an emerging research area is epigenetic regulation of RNA. Over 160 different chemical modifications in RNA have been identified (42). Among them, $\mathrm{N}^{6}$-methyladenosine $\left(\mathrm{m}^{6} \mathrm{~A}\right)$ has been considered the most prevalent modification of RNA Pol II transcripts (43-45). In general, $\mathrm{m}^{6} \mathrm{~A}$ modification is enriched near the $5^{\prime}$ untranslated terminal region (UTR) as well as the stop codon and $3^{\prime}$ UTR, which regulates mRNA transcription (46), splicing (47), export (48), stability (49), and translation $(50,51)$. Like methylation on DNA and histones, $\mathrm{m}^{6} \mathrm{~A}$ is a reversible modification, and fat mass and obesity-associated protein (FTO) is the first RNA demethylase identified for the removal of the methyl group of $\mathrm{m}^{6} \mathrm{~A}$ in mRNA with some extent to $\mathrm{m}^{1} \mathrm{~A}$ in specific tRNAs using $\alpha \mathrm{KG}$ as a co-substrate $(52,53)$.

Accumulating evidence shows that $\mathrm{m}^{6} \mathrm{~A}$ mRNA modification is critical for glioblastoma stem cells self-renewal and tumorigenesis. Though there is some apparent discrepancy in terms of the role of methyltransferase responsible for $\mathrm{m}^{6} \mathrm{~A}$ in glioblastoma (48-50), the consensus is that inhibition of FTO significantly suppresses glioblastoma stem cells on culture and intracranial growth of glioblastoma stem cells in a mouse xenograft model (54-56). Importantly, our recent study has uncovered that D-2HG is a potent inhibitor for the FTO activity in vitro and in vivo, leading to the aberrant accumulation of $\mathrm{m}^{6} \mathrm{~A}$ mRNA in leukemia and glioma cells expressing IDH1 mutant (57). In leukemic cells, FTO inhibition by D-2HG 
decreases stability and thus, expression levels of MYC, one of the master regulators of hyper-anabolism and cell proliferation (57) (Figure 2), though whether this mechanism can be extended to glioma remains to be clarified. The results of our study revealed a surprising functional link between FTO and IDH mutations that could potentially explain why IDH mutated tumors bear proliferative disadvantage.

\section{POTENTIAL IMPACTS OF IDH1 MUTATION ON CELLULAR METABOLISM}

The fact that IDH1 mutant tumors carry a better prognosis than those without IDH1 mutations has led to the hypothesis that this new enzyme may have deleterious effects on cellular metabolism. In accordance with this hypothesis, our study has shown that intracellular metabolism in IDH-mutated glioblastoma is significantly different from that in IDH wild-type glioblastoma, in particular, prominent in nucleotide metabolism pathway (58). To investigate this hypothesis, many investigators have overexpressed the IDHI mutant gene and then performed mass spectroscopy to examine the differences between parental and transformed lines. However, the results so far are mixed and need further studies to interpret the results. Here, we briefly summarize the experimental results, which appear controversial in some cases, and discuss the technical challenge of faithfully recapitulating the IDH1 mutated glioma cellular status.

All studies to date have found that the addition of the IDH1 mutant enzyme comes with a metabolic burden that makes the cell less fit and resilient. The first studies focused on the fact that the IDH1 mutant enzyme was converting large amounts of $\alpha \mathrm{KG}$ into D-2HG, making the assumption that $\alpha \mathrm{KG}$ was derived largely from glutamine. Taking these facts together, it was hypothesized that the IDH1 mutation led to cells becoming glutamine deficient. Seltzer et al. confirmed this by showing that the addition of the IDHI mutant enzyme made cells more vulnerable to glutaminase inhibition (59). Another set of studies focused on the fact that the IDH1 mutant enzyme consumes one molecule of NADPH and produces a molecule of $\mathrm{NADP}^{+}$and, therefore, might have an effect on the level of ROS. Results on this topic have been mixed and sometimes appear controversial. Attempts to knock-in the IDH1 mutation under a Nestin neural stem cell driver were embryonic lethal, but the salvaged cells had lower levels of ROS (60). In contrast, overexpressing the IDH1 mutant enzyme in U87MG cells increased ROS levels and made the cells more vulnerable to radiation (61).

More recently, it was discovered that IDH1 has an important role in reductive carboxylation, which is the ability of the cell to convert glutamine to citrate without going through the TCA cycle. This allows the cell to participate in lipogenesis and membrane synthesis in a hypoxic environment. The presumption would be that with a mutation in the IDH1 enzyme, the native function of the enzyme would be diminished. Again, results have been mixed, with Grassian et al. reporting that overexpression of the IDH1 mutant gene inhibited the ability of cells to perform reductive carboxylation under hypoxia (62). On the other hand, Reitman et al. using the same cell line found that the IDH1 mutation actually facilitated the 
ability of the cell to convert glutamine into fatty acids (palmitate) under hypoxic conditions (63).

\section{A potential caveat and technical challenge to investigate the roles of IDH1 mutation on cellular metabolism}

All of these studies suffer from two methodological problems. The first is that the metabolic effect of the IDH1 mutant enzyme depends on the baseline metabolic background of the host cell. There is increasing evidence that the IDH1 mutation is likely one of the first mutations to occur in gliomagenesis (64) and thus over many rounds of cell growth and selection, the cells have time to adjust and adapt to whatever metabolic effects the IDH1 mutation may have. If the IDH1 mutation were as detrimental to cellular function as studies suggest, the IDH1 mutation would be deleted along with the other genes that impede accelerated cellular growth, for example, PTEN, p53, and NF1. The second is that these studies fail to answer the metabolic effect of blocking the IDH1 mutant enzyme in a glioma cell that already has it.

In order to address these shortcomings, our group performed genetic and metabolic profiling on a panel of patient-derived IDHI wild-type and IDH1 mutant glioblastoma cultures and compared these results with IDH1 mutant overexpression models to determine the accuracy and differences of these models (58). We found that IDH1 wild-type glioblastoma cells had a high genetic expression of de novo nucleotide synthesis genes and disproportionately shunted glucose through the pentose phosphate pathway for de novo nucleotide synthesis. In contrast, IDH1 mutant glioblastoma cells were enriched for DNA repair response genes. Consistent with these predictions, IDH1 wild-type glioblastomas were more vulnerable to de novo nucleotide synthesis inhibitors, and IDH1 mutant glioblastomas were better able to repair DNA after radiation (58), which is also supported by our other studies (65-67). Initially, we assumed that the observed changes in transcriptome and metabolism were due to the direct metabolic effect of IDH mutation. However, surprisingly and importantly, there was no difference seen in nucleotide synthesis when the IDH1 mutant enzyme was overexpressed on an IDH1 wild-type background or when D-2HG production was blocked by an IDH1 mutant inhibitor (58).

Similar to previous studies, overexpression of the IDH1 mutant enzyme depleted TCA cycle intermediates and led to much slower growth (58). In contrast, inhibiting D-2HG formation in endogenous IDH1 mutant cells had no effect on either growth or the level of TCA cycle intermediates. Furthermore, the baseline levels of TCA cycle intermediates were roughly equal between the IDH1 mutant and IDH1 wild-type cultures (58). Taken together, these results suggest that the IDH1 mutation has different effects on different cellular backgrounds and is largely well tolerated in endogenous IDH1 mutant glioma cells. Although IDH1 mutant and IDH1 wild-type gliomas have different metabolic vulnerabilities, these may be largely due to their differences in growth speed and genetic contexts after the long-term gliomagenesis. Further dedicated studies are needed to clarify whether the IDH1 mutation by itself is sufficient to induce the metabolic complexity and heterogeneity of IDH mutated glioma. 


\section{THREE POSSIBLE MODELS EXPLAIN THE APPARENTLY PARADOXICAL OUTCOMES OF IDH1 MUTATION}

Why would a mutation that slows growth be selected over neighboring cells without that mutation and a presumably faster rate of growth? We raise three working models.

\section{Model 1. Increased stress resilience by IDH1 mutation}

One possibility is that the IDH1 mutation enables cells to resist death or antigrowth signals in their microenvironment. This theory is supported by the discovery that the D-2HG molecule could inhibit the function of alphaketoglutarate-dependent enzymes by outcompeting alpha-ketoglutarate (23). his led to the hypothesis that the IDH1 mutation might give a cell the ability to resist environmental influences and prevent differentiation from a progenitor cell to a more differentiated and less prolific cell type. In several cellular contexts, notably fat cells (8), chondrocytes (68) and liver cells (61), overexpressing the IDH1 mutation in precursor/stem cells prevented those cells from differentiating. In each of these previous studies, there was a key mediator gene that was essential for differentiation. During differentiation, this gene was activated by the demethylation of a key histone mark in the promoter or enhancer region. In the presence of the IDH1 mutation or high levels of 2-HG, this histone demethylation was prevented, and the cell failed to differentiate and instead maintained its proliferative potential. However, trying to show that the IDHI mutation has the ability to block differentiation in neural cells has been more elusive. Overexpressing the IDH1 mutation in a mouse sub-ventricular zone (SVZ) stem cell culture changed the default differentiation from a GFAP-positive astrocyte to a TUJ1 positive neuron; however, it did not prevent differentiation or lead to increased growth (8).

\section{Model 2. Chronic malignant evolution via epigenomic repression}

A second possibility is that the effect of the IDH1 mutation is slow but over time can gradually convert the epigenetic state of a cell to a more malignant phenotype. The most popular model of this theory is that the IDH1 mutant enzyme impairs the ability of the TET enzymes to demethylate DNA. In this case, the activity of the DNA methyltransferase (DNMT) enzyme family is unopposed and leads to a gradual increase of methylation throughout the genome. The methylation of CpG islands, particularly in key regulatory regions, tends to decrease the expression of those genes. Over time more and more tumor suppressor genes would become repressed until the cell becomes tumorigenic. The strongest evidence for this theory comes from a study using overexpression of the IDH1 mutant enzyme in an astrocyte line. This led to the gradual induction of Nestin expression and a small increase in growth over many passages. This long-term IDH1 mutant expression was also associated with increased DNA and histone methylation (37). 


\section{Model 3. IDH1 mutation acts as a mutator}

A third possibility is that the IDH1 mutation predisposes to further mutations. When studying patients with IDH1 mutant tumors, it was noted that following resection, when the tumor eventually grew back, the tumors had often acquired a new set of mutations. Mutations that were present in the first tumor were not present in the second tumor. The only mutations that were always present were IDH1 and p53 (64). This result implies that the IDH1 mutation is likely the initial mutation in gliomagenesis and is sufficient to generate enough mutations for tumorigenesis multiple times throughout a patient's life. However, first, p53 must be rendered non-functional. It is not clear how the IDH1 mutation leads to further mutations. One possibility is the methylation and down-regulation of DNA repair machinery, in particular the gene encoding O-6-Methylguanine-DNA Methyltransferase (MGMT), a DNA repair enzyme removing the guanine-alkyl group induced by alkylating agents such as temozolomide. IDH1 mutation and MGMT methylation are correlated, although there are IDH1 mutant tumors with unmethylated MGMT $(69,70)$. As additional evidence, while MGMT methylation is an independent predictor of a positive response to temozolomide in IDH1 wildtype glioma cells, it is not a predictor of chemotherapy response in IDH1 mutant cells, implying that either MGMT itself or the MGMT pathway may be nonfunctional in IDH1 mutant cells (71). Another possibility is that the IDH1 mutation may lead to higher levels of endogenous ROS, predisposing to DNA damage. While presumably the IDH1 mutant enzyme would lead to an alteration in the $\mathrm{NADPH} / \mathrm{NADP}^{+}$equilibrium, it is not obvious a priori what effect this would have on total endogenous ROS levels. Consequently, the question of whether the addition of the IDH1 mutation to cells causes an increase or a decrease in ROS levels is still a matter of debate, with different studies showing conflicting results $(60,61)$.

\section{THERAPEUTIC SENSITIVITY AND RESISTANCE OF IDH MUTANT GLIOMAS}

Standard therapy for a newly diagnosed glioblastoma involves maximal safe surgical resection, temozolomide, and fractionated radiation. This protocol has been validated by randomized controlled trials $(22,23)$. However, these trials were based on a mixed cohort of IDH1 mutant and IDH1 wild-type patients. Presumably, given demographics and prevalence, the majority of these patients were IDH1 wild-type. This means that the results of these trials may not necessarily translate to IDH1 mutant gliomas. IDH1 mutant gliomas are associated with longer survival, and some have assumed that this is due to a better response to adjuvant therapy (temozolomide and radiation) (72). However, arguing against this assumption is the observation that IDH1 wild-type gliomas show increasing therapeutic response and increased survival to higher doses of temozolomide, whereas IDH1 mutant gliomas do not show any improvement with higher doses of temozolomide (73). Due to this concern, additional chemotherapy regimens were tried, and recent trials have shown that CCNU is effective in combination with radiation in IDH1 mutant low-grade gliomas (74). These clinical trials present 
multiple logistical difficulties related to the low incidence of the disease and the relatively long and variable survival.

As many such questions remain unanswered, the most relevant and perhaps most controversial is the question of whether IDHI mutant gliomas are more or less sensitive to radiation than their IDH1 wild-type counterparts. Multiple studies have found conflicting results that seem to depend on the cell line model used and even the culture conditions. Studies using serum culturing conditions found that the IDH1 mutation is associated with increased radiation sensitivity in both overexpression $(61,75)$ and endogenous $(76)$ in vitro studies. In contrast, overexpression (75) and endogenous models (58) grown in serum-free conditions show radiation resistance. There is a shortage of mouse models of IDH1 mutant gliomas; however, in one of the few studies to utilize a mouse model, the IDH1 mutation was associated with radiation resistance via upregulation of DNA damage response genes (77). The general clinical consensus is that radiation is effective against IDH1 mutant gliomas, and no randomized clinical trial is to test this assertion is forthcoming.

\section{Small molecule IDH1 mutant inhibitor}

Following the discovery of the IDH1 mutation, there was a great deal of interest in developing mutant IDH targeted therapy, leading to a series of potent small molecule inhibitors against mutant IDH1 (for example, AGI-5198) and IDH2 enzyme (e.g., AGI-6780) (Figure 4) (78). In the case of the leukemia model TF-1, mutant IDH2 specific inhibitor AGI-6780 prevented the changes seen following IDH2 mutant expression and induced differentiation of the IDH2 mutated leukemic cells (79). Consistent with these findings, early clinical trials with similar inhibitors in acute myeloid leukemia are also promising (80). After taking the IDH1 mutant inhibitor, patients with IDH1 mutant acute myeloid leukemia show a progressive decrease in the number of immature tumor-type myeloid cells with a corresponding increase in mature differentiated cells. Most encouragingly, unlike traditional chemotherapy, there is no myelosuppression seen across the other myeloid lineages.

However, in the case of IDH1 mutant glioma models, the results were more mixed. The first attempt to treat an IDH1 mutant glioma with the inhibitor was met with some success. Treatment of mutant IDHl inhibitor AGI-5198, the first prototype inhibitor $(78,81)$, decreased glioma size and increased expression of GFAP, suggesting differentiation (82). However, later attempts to repeat this data have failed. In one of the more thorough studies, Tateishi et al. treated IDHI mutant cells with AGI-5198 for over a year and found no difference in either DNA methylation or histone modification, and there was a slight increase in growth with the addition of AGI-5198 (83). These variable results from the preclinical studies are, in part, likely due to the poor metabolic stability and low blood-brain barrier penetrance of the compound (78).

One difficulty with mutant IDHI inhibitor to treat brain tumor models is the issue of time. While Turcan et al. was able to demonstrate an increase in methylation with the addition of the IDH1 mutant enzyme, the effect required the cells to undergo 40 passages and presumably several hundred cell divisions (37). Even then, the majority of the newly methylated sites were only partially methylated. 


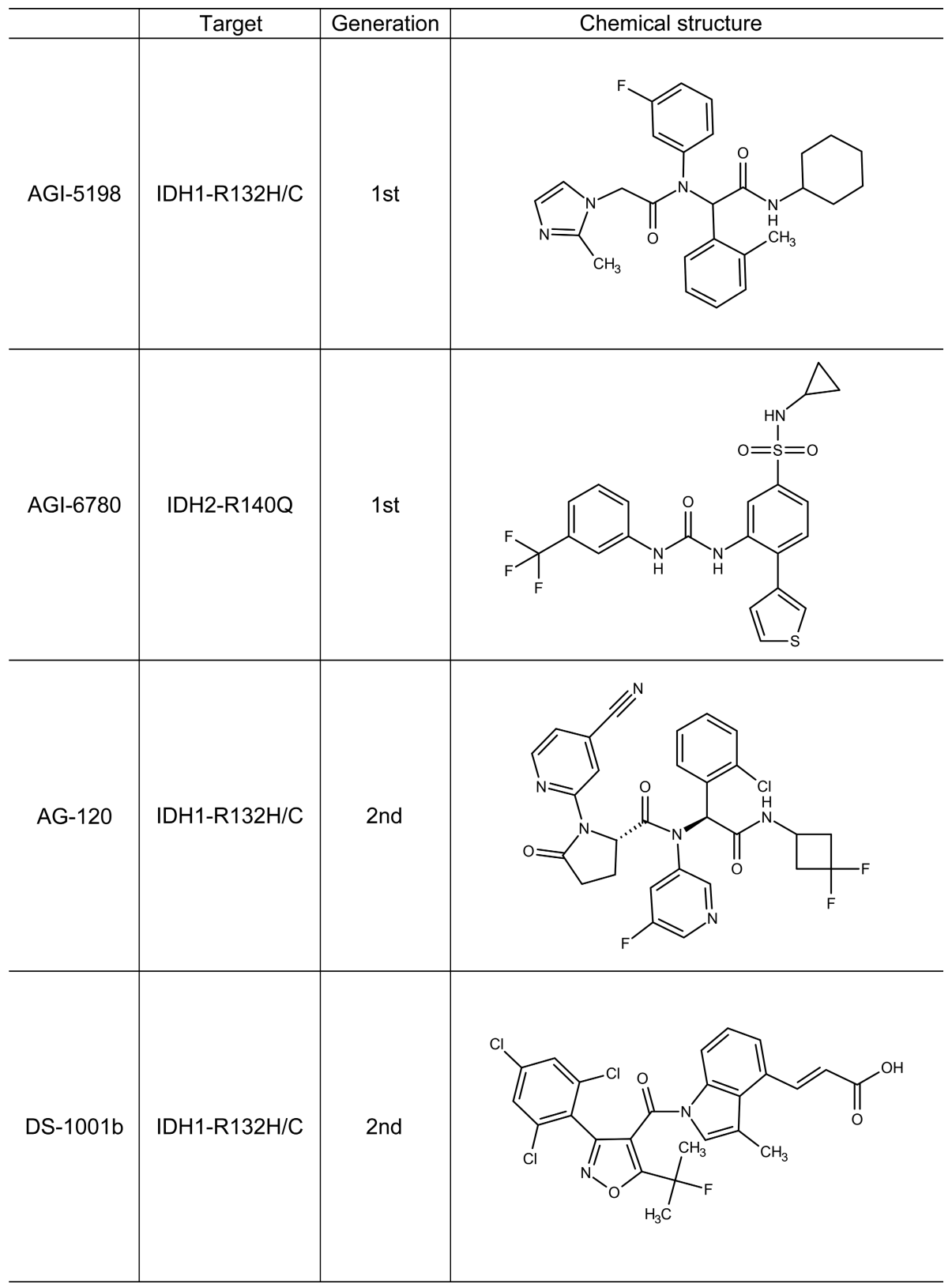

Figure 4. Potential molecules for treatment of IDH mutated cancers. The chemical structure of inhibitors of IDH mutants are shown. AGI-5198, AG-120, and DS-1001b target the IDH1 mutants (R132H and R132C), and AGI-6780 targets an IDH2 mutant (R140Q). AG-120 and DS-1001b are expected as the next-generation therapeutics for curing glioblastoma. 
Any study that fails to find an effect of adding the IDH1 mutant enzyme can be criticized for not giving the cells enough time no matter how much time was given. One possibility for the disagreement between the two studies is that while the IDH1 mutation is able to induce methylation, once the methylation is induced, it is irreversible. It is also difficult to translate these results into a clinical context where the vast majority of cells in the brain are post-mitotic.

Nevertheless, given the potential for targeted efficacy with limited off-target toxicity, many IDHl mutant inhibitors have entered clinical trials. AG-120/ Ivosidenib is showing a good safety profile and a trend for tumor stability in non-enhancing tumors (84) (Clinical Trials: NCT02073994; NCT03343197). Furthermore, clinical trials with another next-generation compound with greater blood-brain barrier penetration, DS-1001b (85), are currently enrolling for glioma patients with an IDH1 mutation (NCT03030066; NCT04458272) (Figure 4).

\section{CONCLUSION}

In this chapter, we introduced the impact of wild-type and mutant IDHI on glioblastoma metabolism, growth, and current therapeutic approach. Since its discovery nearly a decade ago, the IDHI mutation has fast become one of the most wellknown and complicated metabolic mutations found in cancer. Convincing evidence exists that it is the initial mutation that begins the process of tumorigenesis. Despite the difficulty of modeling its behavior in vitro, significant strides have been made to link the derangement in metabolic function to its deregulation of epigenetics and, finally, its effect on growth. Overexpression models of IDH1 mutant function likely over-estimate the negative effects of the mutant enzyme on growth and metabolic function. In several studies, inhibiting the IDH1 mutant enzyme in endogenous cultures seems to have minimal effects on either growth or the metabolic state of the cell. Our study also demonstrates the tumor-suppressive effect of the accumulated D-2HG by FTO inhibition. However, all studies demonstrate IDH1 mutant and IDH1 wild-type gliomas have different metabolic properties, pointing that they may have distinctive vulnerabilities allowing for the possibility of personalized therapy. Collectively, these results suggest that further and broader investigation of the mechanistic role of these enzymes in IDH1-wild-type and mutant glioma is warranted.

Acknowledgment:This work has been supported by the MTP UC-Brain Tumor Center grant, Ohio Cancer Research, B*Cured, JSPS KAKENHI (20H03165), R21NS100077, and R01NS089815 (A.T.S.), and the Fund for the Promotion of Joint International Research (Fostering Joint International Research (A)) from the JSPS, 18KK0455 (Y.H.). This work was supported in part by research funds from the Yamagata prefectural government and the City of Tsuruoka.

Conflict of interest: The authors declare no potential conflicts of interest with respect to research, authorship, and/or publication of this chapter.

Copyright and Permission Statement: The authors confirm that the materials included in this chapter do not violate copyright laws. Where relevant, appropriate permissions have been obtained from the original copyright holder(s), and all original sources have been appropriately acknowledged or referenced. 


\section{REFERENCES}

1. Ohgaki H, Kleihues P. Genetic pathways to primary and secondary glioblastoma. Am J Pathol. 2007;170(5):1445-53. https://doi.org/10.2353/ajpath.2007.070011

2. Parsons DW, Jones S, Zhang X, Lin JC-H, Leary RJ, Angenendt P, et al. An integrated genomic analysis of human glioblastoma multiforme. Science 2008;321(5897):1807-12. https://doi.org/10.1126/ science. 1164382

3. Yan H, Parsons DW, Jin G, McLendon R, Rasheed BA, Yuan W, et al. IDHl and IDH2 mutations in gliomas. N Engl J Med. 2009;360(8):765-73. https://doi.org/10.1056/NEJMoa0808710

4. Molenaar RJ, Verbaan D, Lamba S, Zanon C, Jeuken JWM, Boots-Sprenger SHE, et al. The combination of IDHl mutations and MGMT methylation status predicts survival in glioblastoma better than either IDHl or MGMT alone. Neuro Oncol. 2014;16(9):1263-73. https://doi.org/10.1093/neuonc/ nou005

5. Dang L, White DW, Gross S, Bennett BD, Bittinger MA, Driggers EM, et al. Cancer-associated IDHI mutations produce 2-hydroxyglutarate. Nature. 2009;462(7274):739-44. https://doi.org/10.1038/ nature 08617

6. Hartmann C, Meyer J, Balss J, Capper D, Mueller W, Christians A, et al. Type and frequency of IDH1 and IDH2 mutations are related to astrocytic and oligodendroglial differentiation and age: a study of 1,010 diffuse gliomas. Acta Neuropathol. 2009;118(4):469-74. https://doi.org/10.1007/ s00401-009-0561-9

7. Sonoda Y, Kumabe T, Nakamura T, Saito R, Kanamori M, Yamashita Y, et al. Analysis of IDHl and IDH2 mutations in Japanese glioma patients. Cancer Sci. 2009;100(10):1996-8. https://doi. org/10.1111/j.1349-7006.2009.01270.x

8. Lu C, Ward PS, Kapoor GS, Rohle D, Turcan S, Abdel-Wahab O, et al. IDH mutation impairs histone demethylation and results in a block to cell differentiation. Nature. 2012;483(7390):474-8. https:// doi.org/10.1038/nature10860

9. Losman J-A, Kaelin WG. What a difference a hydroxyl makes: mutant IDH, (R)-2-hydroxyglutarate, and cancer. Genes Dev. 2013;27(8):836-52. https://doi.org/10.1101/gad.217406.113

10. Flavahan WA, Drier Y, Liau BB, Gillespie SM, Venteicher AS, Stemmer-Rachamimov AO, et al. Insulator dysfunction and oncogene activation in IDH mutant gliomas. Nature. 2016;529(7584): 110-4. https://doi.org/10.1038/nature 16490

11. Cancer Genome Atlas Research Network, Brat DJ, Verhaak RGW, Aldape KD, Yung WKA, Salama SR, et al. Comprehensive, Integrative Genomic Analysis of Diffuse Lower-Grade Gliomas. N Engl J Med. 2015;372(26):2481-98. https://doi.org/10.1056/NEJMoal402121

12. Ramachandran N, Colman RF. Chemical characterization of distinct subunits of pig heart DPNspecific isocitrate dehydrogenase. J Biol Chem. 1980;255(18):8859-64. https://doi.org/10.1016/ S0021-9258(18)43581-8

13. Gabriel JL, Zervos PR, Plaut GW. Activity of purified NAD-specific isocitrate dehydrogenase at modulator and substrate concentrations approximating conditions in mitochondria. Metabolism. 1986;35(7):661-7. https://doi.org/10.1016/0026-0495(86)90175-7

14. Stoddard BL, Dean A, Koshland DE. Structure of isocitrate dehydrogenase with isocitrate, nicotinamide adenine dinucleotide phosphate, and calcium at 2.5-A resolution: a pseudo-Michaelis ternary complex. Biochemistry. 1993;32(36):9310-6. https://doi.org/10.1021/bi00087a008

15. Wise DR, Ward PS, Shay JES, Cross JR, Gruber JJ, Sachdeva UM, et al. Hypoxia promotes isocitrate dehydrogenase-dependent carboxylation of $\alpha$-ketoglutarate to citrate to support cell growth and viability. Proc Natl Acad Sci U S A. 2011;108(49):19611-6. https://doi.org/10.1073/ pnas. 1117773108

16. Mullen AR, Wheaton WW, Jin ES, Chen P-H, Sullivan LB, Cheng T, et al. Reductive carboxylation supports growth in tumour cells with defective mitochondria. Nature. 2011;481(7381):385-8. https://doi.org/10.1038/nature10642

17. Tan F, Jiang Y, Sun N, Chen Z, Lv Y, Shao K, et al. Identification of isocitrate dehydrogenase 1 as a potential diagnostic and prognostic biomarker for non-small cell lung cancer by proteomic analysis. Mol Cell Proteomics. 2012;11(2):M111.008821. https://doi.org/10.1074/mcp.M111.008821 
18. Al-Amodi HSAB, Nabih ES, Kamel HFM, Sayed El MA, Dwedar IAM. Wild-Type Isocitrate Dehydrogenase 1 Over-Expression is Related to Cancer Stem Cells Survival in Lung Adenocarcinoma. Cancer Invest. 2018;36(3):185-9. https://doi.org/10.1080/07357907.2018.1445262

19. Zarei M, Lal S, Parker SJ, Nevler A, Vaziri-Gohar A, Dukleska K, et al. Posttranscriptional Upregulation of IDHl by HuR Establishes a Powerful Survival Phenotype in Pancreatic Cancer Cells. Cancer Res. 2017;77(16):4460-71. https://doi.org/10.1158/0008-5472.CAN-17-0015

20. Wahl DR, Dresser J, Wilder-Romans K, Parsels JD, Zhao SG, Davis M, et al. Glioblastoma Therapy Can Be Augmented by Targeting IDH1-Mediated NADPH Biosynthesis. Cancer Res. 2017;77(4):960-70. https://doi.org/10.1158/0008-5472.CAN-16-2008

21. Calvert AE, Chalastanis A, Wu Y, Hurley LA, Kouri FM, Bi Y, et al. Cancer-Associated IDH1 Promotes Growth and Resistance to Targeted Therapies in the Absence of Mutation. Cell Rep. 2017;19(9): 1858-73. https://doi.org/10.1016/j.celrep.2017.05.014

22. Stupp R, Hegi ME, Mason WP, van den Bent MJ, Taphoorn MJ, Janzer RC, et al. Effects of radiotherapy with concomitant and adjuvant temozolomide versus radiotherapy alone on survival in glioblastoma in a randomised phase III study: 5-year analysis of the EORTC-NCIC trial. Lancet Oncol. 2009;10(5):459-66. https://doi.org/10.1016/S1470-2045(09)70025-7

23. Stupp R, Mason WP, Van Den Bent MJ, Weller M, Fisher B, Taphoorn MJB, et al. Radiotherapy plus concomitant and adjuvant temozolomide for glioblastoma. N Engl J Med. 2005;352(10):987-96. https://doi.org/10.1056/NEJMoa043330

24. Stupp R, Taillibert S, Kanner A, Read W, Steinberg D, Lhermitte B, et al. Effect of Tumor-Treating Fields Plus Maintenance Temozolomide vs Maintenance Temozolomide Alone on Survival in Patients With Glioblastoma: A Randomized Clinical Trial. JAMA. 2017;318(23):2306-16. https://doi.org/10.1001/ jama.2017.18718

25. Bergaggio E, Piva R. Wild-Type IDH Enzymes as Actionable Targets for Cancer Therapy. Cancers. 2019;11(4):563. https://doi.org/10.3390/cancers11040563

26. Ward JF. Biochemistry of DNA lesions. Radiat Res Suppl. 1985;8:S103-11. https://doi.org/10.2307/ 3583517

27. Powell S, McMillan TJ. DNA damage and repair following treatment with ionizing radiation. Radiother Oncol. 1990;19(2):95-108. https://doi.org/10.1016/0167-8140(90)90123-E

28. Zhou W, Wahl DR. Metabolic Abnormalities in Glioblastoma and Metabolic Strategies to Overcome Treatment Resistance. Cancers. 2019;11(9):1231. https://doi.org/10.3390/cancers11091231

29. Kim SY, Yoo YH, Park J-W. Silencing of mitochondrial NADP(+)-dependent isocitrate dehydrogenase gene enhances glioma radiosensitivity. Biochem and Biophys Res Commun. 2013;433(2):260-5. https://doi.org/10.1016/j.bbrc.2013.02.093

30. Cappetta D, De Angelis A, Sapio L, Prezioso L, Illiano M, Quaini F, et al. Oxidative Stress and Cellular Response to Doxorubicin: A Common Factor in the Complex Milieu of Anthracycline Cardiotoxicity. Oxid Med Cell Longev. Hindawi; 2017;2017(16):1521020-13. https://doi.org/10.1155/2017/1521020

31. Gerweck LE, Wakimoto H. At the Crossroads of Cancer Stem Cells, Radiation Biology, and Radiation Oncology. Cancer Res. 2016;76(5):994-8. https://doi.org/10.1158/0008-5472.CAN-15-2455

32. Cloughesy TF, Cavenee WK, Mischel PS. Glioblastoma: From Molecular Pathology to Targeted Treatment. Annu Rev Pathol. 2014;9(1):1-25. https://doi.org/10.1146/annurev-pathol-011110-130324

33. Nakano I. Stem cell signature in glioblastoma: therapeutic development for a moving target. J Neurosurg. 2014;14:324-30. https://doi.org/10.3171/2014.9.JNS132253

34. Lathia JD, Mack SC, Mulkearns-Hubert EE, Valentim CLL, Rich JN. Cancer stem cells in glioblastoma. Genes Dev. 2015;29(12):1203-17. https://doi.org/10.1101/gad.261982.115

35. Sjöblom T, Jones S, Wood LD, Parsons DW, Lin J, Barber TD, et al. The consensus coding sequences of human breast and colorectal cancers. Science. 2006;314(5797):268-74. https://doi.org/10.1126/ science. 1133427

36. Shi J, Zuo H, Ni L, Xia L, Zhao L, Gong M, et al. An IDHl mutation inhibits growth of glioma cells via GSH depletion and ROS generation. Neurol Sci. 2014;35(6):839-45. https://doi.org/10.1007/ s10072-013-1607-2

37. Turcan S, Rohle D, Goenka A, Walsh LA, Fang F, Yilmaz E, et al. IDHl mutation is sufficient to establish the glioma hypermethylator phenotype. Nature. 2012;483(7390):479-83. https://doi.org/ 10.1038/nature10866 
38. Figueroa ME, Abdel-Wahab O, Lu C, Ward PS, Patel J, Shih A, et al. Leukemic IDH1 and IDH2 mutations result in a hypermethylation phenotype, disrupt TET2 function, and impair hematopoietic differentiation. Cancer Cell. 2010;18(6):553-67. https://doi.org/10.1016/j.ccr.2010.11.015

39. Xu W, Yang H, Liu Y, Yang Y, Wang P, Kim S-H, et al. Oncometabolite 2-hydroxyglutarate is a competitive inhibitor of $\alpha$-ketoglutarate-dependent dioxygenases. Cancer Cell. 2011;19(1):17-30. https:// doi.org/10.1016/j.ccr.2010.12.014

40. Chowdhury R, Yeoh KK, Tian Y-M, Hillringhaus L, Bagg EA, Rose NR, et al. The oncometabolite 2-hydroxyglutarate inhibits histone lysine demethylases. EMBO Rep. 2011;12(5):463-9. https://doi. org/10.1038/embor.2011.43

41. Janke R, Iavarone AT, Rine J. Oncometabolite D-2-Hydroxyglutarate enhances gene silencing through inhibition of specific H3K36 histone demethylases. Elife. 2017;6:35. https://doi.org/10.7554/eLife.22451

42. Boccaletto P, Machnicka MA, Purta E, Piatkowski P, Baginski B, Wirecki TK, et al. MODOMICS: a database of RNA modification pathways. 2017 update. Nucleic Acids Research. 2018;46(D1):D303-7. https://doi.org/10.1093/nar/gkx1030

43. Desrosiers R, Friderici K, Rottman F. Identification of methylated nucleosides in messenger RNA from Novikoff hepatoma cells. Proc Natl Acad Sci U S A. 1974;71(10):3971-5. https://doi.org/10.1073/ pnas.71.10.3971

44. Alarcón CR, Lee H, Goodarzi H, Halberg N, Tavazoie SF. N6-methyladenosine marks primary microRNAs for processing. Nature. 2015;519(7544):482-5. https://doi.org/10.1038/nature14281

45. Patil DP, Chen C-K, Pickering BF, Chow A, Jackson C, Guttman M, et al. m(6)A RNA methylation promotes XIST-mediated transcriptional repression. Nature. 2016;537(7620):369-73. https://doi. org/10.1038/nature 19342

46. Liu J, Dou X, Chen C, Chen C, Liu C, Xu MM, et al. N6-methyladenosine of chromosome-associated regulatory RNA regulates chromatin state and transcription. Science. 2020;367(6477):580-6. https:// doi.org/10.1126/science.aay6018

47. Xiao W, Adhikari S, Dahal U, Chen Y-S, Hao Y-J, Sun B-F, et al. Nuclear m(6)A Reader YTHDCl Regulates mRNA Splicing. Mol Cell. 2016;61(4):507-19. https://doi.org/10.1016/j.molcel.2016.01.012

48. Roundtree IA, Luo G-Z, Zhang Z, Wang X, Zhou T, Cui Y, et al. YTHDCl mediates nuclear export of N6-methyladenosine methylated mRNAs. Elife. 2017;6:1299. https://doi.org/10.7554/eLife.31311

49. Wang X, Lu Z, Gomez A, Hon GC, Yue Y, Han D, et al. N6-methyladenosine-dependent regulation of messenger RNA stability. Nature. 2014;505(7481):117-20. https://doi.org/10.1038/nature12730

50. Wang X, Zhao BS, Roundtree IA, Lu Z, Han D, Ma H, et al. N(6)-methyladenosine Modulates Messenger RNA Translation Efficiency. Cell. 2015;161(6):1388-99. https://doi.org/10.1016/j.cell.2015.05.014

51. Zhao BS, Roundtree IA, He C. Post-transcriptional gene regulation by mRNA modifications. Nat Rev Mol Cell Biol. 2017;18(1):31-42. https://doi.org/10.1038/nrm.2016.132

52. Wei J, Liu F, Lu Z, Fei Q, Ai Y, He PC, et al. Differential m6A, m6Am, and mlA Demethylation Mediated by FTO in the Cell Nucleus and Cytoplasm. Mol Cell. 2018;71(6):973-5. https://doi. org/10.1016/j.molcel.2018.08.011

53. Shi H, Wei J, He C. Where, When, and How: Context-Dependent Functions of RNA Methylation Writers, Readers, and Erasers. Mol Cell. 2019;74(4):640-50. https://doi.org/10.1016/j.molcel.2019.04.025

54. Cui Q, Shi H, Ye P, Li L, Qu Q, Sun G, et al. m6A RNA Methylation Regulates the Self-Renewal and Tumorigenesis of Glioblastoma Stem Cells. Cell Rep. 2017;18(11):2622-34. https://doi.org/10.1016/j. celrep.2017.02.059

55. Xiao L, Li X, Mu Z, Zhou J, Zhou P, Xie C, et al. FTO Inhibition Enhances the Antitumor Effect of Temozolomide by Targeting MYC-miR-155/23a Cluster-MXI1 Feedback Circuit in Glioma. Cancer Res. 2020;80(18):3945-58. https://doi.org/10.1158/0008-5472.CAN-20-0132

56. Galardi S, Michienzi A, Ciafrè SA. Insights into the Regulatory Role of m6A Epitranscriptome in Glioblastoma. IJMS. 2020;21(8):2816. https://doi.org/10.3390/ijms21082816

57. Su R, Dong L, Li C, Nachtergaele S, Wunderlich M, Qing Y, et al. R-2HG Exhibits Anti-tumor Activity by Targeting FTO/m6A/MYC/CEBPA Signaling. Cell. 2018;172(1-2):90-91.e23. https://doi. org/10.1016/j.cell.2017.11.031

58. Garrett M, Sperry J, Braas D, Yan W, Le TM, Mottahedeh J, et al. Metabolic characterization of isocitrate dehydrogenase (IDH) mutant and IDH wildtype gliomaspheres uncovers cell type-specific vulnerabilities. Cancer Metab. 2018;6(1):4. https://doi.org/10.1186/s40170-018-0177-4 
59. Seltzer MJ, Bennett BD, Joshi AD, Gao P, Thomas AG, Ferraris DV, et al. Inhibition of glutaminase preferentially slows growth of glioma cells with mutant IDH1. Cancer Res. 2010;70(22):8981-7. https:// doi.org/10.1158/0008-5472.CAN-10-1666

60. Sasaki M, Knobbe CB, Itsumi M, Elia AJ, Harris IS, Chio IIC, et al. D-2-hydroxyglutarate produced by mutant IDHl perturbs collagen maturation and basement membrane function. Genes Dev. 2012;26(18):2038-49. https://doi.org/10.1101/gad.198200.112

61. Li S, Chou AP, Chen W, Chen R, Deng Y, Phillips HS, et al. Overexpression of isocitrate dehydrogenase mutant proteins renders glioma cells more sensitive to radiation. Neuro Oncol. 2013;15(1):57-68. https://doi.org/10.1093/neuonc/nos261

62. Grassian AR, Parker SJ, Davidson SM, Divakaruni AS, Green CR, Zhang X, et al. IDHl mutations alter citric acid cycle metabolism and increase dependence on oxidative mitochondrial metabolism. Cancer Res. 2014;74(12):3317-31. https://doi.org/10.1158/0008-5472.CAN-14-0772-T

63. Reitman ZJ, Duncan CG, Poteet E, Winters A, Yan L-J, Gooden DM, et al. Cancer-associated isocitrate dehydrogenase 1 (IDH1) R132H mutation and d-2-hydroxyglutarate stimulate glutamine metabolism under hypoxia. J Biol Chem. 2014;289(34):23318-28. https://doi.org/10.1074/jbc.M114.575183

64. Johnson BE, Mazor T, Hong C, Barnes M, Aihara K, McLean CY, et al. Mutational analysis reveals the origin and therapy-driven evolution of recurrent glioma. Science. 2014;343(6167):189-93. https:// doi.org/10.1126/science.1239947

65. Kofuji S, Hirayama A, Eberhardt AO, Kawaguchi R, Sugiura Y, Sampetrean O, et al. IMP dehydrogenase-2 drives aberrant nucleolar activity and promotes tumorigenesis in glioblastoma. Nat Cell Biol. 2019;21(8):1-18. https://doi.org/10.1038/s41556-019-0363-9

66. Kofuji S, Sasaki AT. GTP Metabolic Reprogramming by IMPDH2: Unlocking Cancer Cells' Fueling Mechanism. J. Biochem. 2020;168(4):319-28. https://doi.org/10.1093/jb/mvaa085

67. Wang X, Yang K, Xie Q, Wu Q, Mack SC, Shi Y, et al. Purine synthesis promotes maintenance of brain tumor initiating cells in glioma. Nat Neurosci. 2017;20(5):661-73. https://doi.org/10.1038/nn.4537

68. Suijker J, Oosting J, Koornneef A, Struys EA, Salomons GS, Schaap FG, et al. Inhibition of mutant IDH1 decreases D-2-HG levels without affecting tumorigenic properties of chondrosarcoma cell lines. Oncotarget. 2015;6(14):12505-19. https://doi.org/10.18632/oncotarget.3723

69. Tuononen K, Tynninen O, Sarhadi VK, Tyybäkinoja A, Lindlöf M, Antikainen M, et al. The hypermethylation of the O6-methylguanine-DNA methyltransferase gene promoter in gliomas--correlation with array comparative genome hybridization results and IDHl mutation. Genes Chromosomes Cancer. 2012;51(1):20-9. https://doi.org/10.1002/gcc.20927

70. Leu S, Felten von S, Frank S, Boulay J-L, Mariani L. IDH mutation is associated with higher risk of malignant transformation in low-grade glioma. J Neurooncol. 2016;127(2):363-72. https://doi. org/10.1007/s11060-015-2048-y

71. Wick W, Meisner C, Hentschel B, Platten M, Schilling A, Wiestler B, et al. Prognostic or predictive value of MGMT promoter methylation in gliomas depends on IDH1 mutation. Neurology. 2013;81(17):1515-22. https://doi.org/10.1212/WNL.0b013e3182a95680

72. SongTao Q, Lei Y, Si G, YanQing D, HuiXia H, XueLin Z, et al. IDH mutations predict longer survival and response to temozolomide in secondary glioblastoma. Cancer Sci. 2012;103(2):269-73. https:// doi.org/10.1111/j.1349-7006.2011.02134.x

73. Verhaak RGW, Hoadley KA, Purdom E, Wang V, Qi Y, Wilkerson MD, et al. Integrated genomic analysis identifies clinically relevant subtypes of glioblastoma characterized by abnormalities in PDGFRA, IDH1, EGFR, and NF1. Cancer Cell 2010;17(1):98-110.

74. Bell EH, Zhang P, Shaw EG, Buckner JC, Barger GR, Bullard DE, et al. Comprehensive Genomic Analysis in NRG Oncology/RTOG 9802: A Phase III Trial of Radiation Versus Radiation Plus Procarbazine, Lomustine (CCNU), and Vincristine in High-Risk Low-Grade Glioma. J Clin Oncol. 2020;38(29):3407-17. https://doi.org/10.1200/JCO.19.02983

75. Yin N, Xie T, Zhang H, Chen J, Yu J, Liu F. IDH1-R132H mutation radiosensitizes U87MG glioma cells via epigenetic downregulation of TIGAR. Oncol Lett. 2020;19(2):1322-30. https://doi.org/10.3892/ ol.2019.11148

76. Sulkowski PL, Corso CD, Robinson ND, Scanlon SE, Purshouse KR, Bai H, et al. 2-Hydroxyglutarate produced by neomorphic IDH mutations suppresses homologous recombination and induces PARP inhibitor sensitivity. Sci Transl Med. 2017;9(375):eaal2463-32. https://doi.org/10.1126/scitranslmed.aal2463 
77. Nunez FJ, Mendez FM, Kadiyala P, Alghamri MS, Savelieff MG, Garcia-Fabiani MB, et al. IDH1-R132H acts as a tumor suppressor in glioma via epigenetic up-regulation of the DNA damage response. Science Transl Med. 2019;11(479). https://doi.org/10.1126/scitranslmed.aaq1427

78. Dang L, Su S-SM. Isocitrate Dehydrogenase Mutation and (R)-2-Hydroxyglutarate: From Basic Discovery to Therapeutics Development. Annu Rev Biochem. 2017;86(1):305-31. https://doi.org/ 10.1146/annurev-biochem-061516-044732

79. Wang F, Travins J, DeLaBarre B, Penard-Lacronique V, Schalm S, Hansen E, et al. Targeted inhibition of mutant IDH2 in leukemia cells induces cellular differentiation. Science. 2013;340(6132):622-6. https://doi.org/10.1126/science. 1234769

80. IDHl inhibitor shows promising early results. Cancer Discov. 2015;4-4. https://doi.org/10.1158/21598290.CD-NB2015-165

81. Popovici-Muller J, Saunders JO, Salituro FG, Travins JM, Yan S, Zhao F, et al. Discovery of the First Potent Inhibitors of Mutant IDH1 That Lower Tumor 2-HG in Vivo. ACS Med Chem Lett. 2012;3(10):850-5. https://doi.org/10.1021/ml300225h

82. Rohle D, Popovici-Muller J, Palaskas N, Turcan S, Grommes C, Campos C, et al. An inhibitor of mutant IDHl delays growth and promotes differentiation of glioma cells. Science. 2013;340(6132):626-30. https://doi.org/10.1126/science. 1236062

83. Tateishi K, Wakimoto H, Iafrate AJ, Tanaka S, Loebel F, Lelic N, et al. Extreme Vulnerability of IDHI Mutant Cancers to NAD+ Depletion. Cancer Cell. 2015;28(6):773-84. https://doi.org/10.1016/j. ccell.2015.11.006

84. Mellinghoff IK, Ellingson BM, Touat M, Maher E, La Fuente De MI, Holdhoff M, et al. Ivosidenib in Isocitrate Dehydrogenase 1-Mutated Advanced Glioma. J Clin Oncol. 2020;38(29):3398-406. https://doi.org/10.1200/JCO.19.03327

85. Machida Y, Nakagawa M, Matsunaga H, Yamaguchi M, Ogawara Y, Shima Y, et al. A Potent BloodBrain Barrier-Permeable Mutant IDHI Inhibitor Suppresses the Growth of Glioblastoma with IDH1 Mutation in a Patient-Derived Orthotopic Xenograft Model. Mol Cancer Ther. 2020;19(2):375-83. https://doi.org/10.1158/1535-7163.MCT-18-1349 